\title{
The efficacy of functional supports in mediating the effects of exercise on shoulder joint position sense
}

\author{
Lynsey M. Wilson and Matt Greig* \\ Sports Injuries Research Group, Department of Sport and Physical Activity, Edge Hill University, Ormskirk, UK
}

Received 15 July 2016

Accepted 14 December 2016

\section{Abstract.}

BACKGROUND: Prolonged exercise and joint position sense are considered aetiological risk factors for shoulder injury in overhead sports.

OBJECTIVE: To investigate the efficacy of a neoprene sleeve and kinesiology tape (KT) in mediating the effect of exercise on shoulder joint position sense.

METHODS: 25 overhead sports participants (women - 10, men - 15) completed 30 maximal isokinetic repetitions at $120^{\circ} / \mathrm{s}$ of three shoulder exercise protocols: 9090 (seated $90^{\circ}$ shoulder abduction, $90^{\circ}$ elbow flexion, modified neutral (seated $45^{\circ}$ shoulder abduction, $90^{\circ}$ elbow flexion in the scapular plane and diagonal (seated GHJ flexion, abduction, external rotation and extension, adduction and internal rotation. Absolute error in active reproduction of passive positioning was assessed pre- and post-exercise at $10 \%$ s. This was conducted for end range internal and external rotation, and mid-range.

RESULTS: A repeated measures general linear model revealed no significant main effect for gender or exercise in any test position. A main effect for support condition was observed pre-exercise in the KT condition, with JPS significantly $(p<0.01)$ impaired $\left(5.30 \pm 2.16^{\circ}\right)$ relative to baseline control scores $\left(4.11 \pm 2.81^{\circ}\right)$ in end range external rotation at the 9090 position. CONCLUSION: Results indicated that neither neoprene nor kinesio-tape were more effective in limiting the effects of exercise on joint position sense (JPS). Healthy, un-injured overhead athletes may not need to consider taping or supportive device, indeed KT application was observed to impair JPS in specific movements.

Keywords: Joint position sense, kinesiology tape, neoprene sleeve, exercise, shoulder

\section{Introduction}

In overhead sports such as tennis, badminton, volleyball and cricket (bowling) extended and prolonged play is often necessary. It has been suggested that this type of repetitive and prolonged exercise may alter the normal kinematics of the gleno-humeral joint (GHJ) from a joint position sense (JPS) perspective [1]. As such it may be considered as an aetiological risk fac-

${ }^{*}$ Corresponding author: Matt Greig, Sports Injuries Research Group, Department of Sport and Physical Activity, Edge Hill University, Ormskirk L39 4QP, UK. Tel.: +44 01695 584848; Fax: +44 0 1695 584812; E-mail:matt.greig@edgehill.ac.uk. tor. Such an intrinsic risk factor may leave an athlete susceptible to a reduction in performance or indeed an increased occurrence of injury. Changes in muscle activity and function of the mechanoreceptors may be associated with injury and loss of performance as deficits in JPS may lead to failure of the rotator cuff (RC) to control stability during movement [2]. Therefore, alterations to mechanoreceptor efficacy may have a detrimental effect.

Functional instability within the GHJ may be linked to decreased proprioception and alterations in neuromuscular control [3] suggesting that alterations to JPS and afferent pathways may increase susceptibility to injury. Alterations to mechanoreceptor efficacy may 
have a detrimental effect on performance. Joint position sense (JPS) acuity is influenced by ranges of motion at a joint [4]. Similarly, there is a decrease in mechanoreceptor stimuli when the GHJ is in a lax, mid-joint position [5]. The influence of prolonged exercise on the dynamic stabilisation of the rotator cuff may be considered as an aetiological risk factor and as such extrapolated to sports requiring repetitive GHJ motions. Many recreational and amateur level athletes will strive to utilise the most effective methods to overcome and offset the negative effects of game play on the GHJ. This may be from an extrinsic aspect in the form of functional supports such as neoprene sleeves and taping such as Kinesio-tape (KT).

Only a few studies have examined the effects of supportive devices or supports on the GHJ. Conflicting results have been proposed determining that a neoprene shoulder sleeve positively affected JPS errors scores measured isokinetically [6], whilst in contrast no positive decrease in error scores when wearing a neoprene shoulder stabiliser. Indeed, for positions of mid and end of range external rotation recorded higher and hence poorer JPS error scores whilst wearing the support [7]. The effects of KT on shoulder JPS has not been examined. However, no increase in static proprioception was found on ankle JPS when compared to no taping in a group of healthy individuals [8]. The ability for an athlete to undertake their chosen sport whilst limiting the negative impact of prolonged exercise is of benefit. Supports such as neoprene sleeves and kinesio-tape may help to control and mediate the effects of prolonged exercise on GHJ structures thus maintaining stability.

This study aimed to assess the effects of an isokinetically induced exercise protocol and the application of shoulder supportive devices on joint position sense by examining active reproduction of passive positioning (ARPP) [9] of the dominant arm GHJ of athletes involved in overhead sports. The study aimed to assess ARPP angles through a functional range of motion. A comparison of the efficacy of a functional support (a neoprene shoulder sleeve) in relation to a KT functional taping technique was evaluated. The study tailored the exercise protocol to mimic the requirements of overhead sports, therefore, generating results applicable to game duration.

\section{Method \\ 2.1. Participants}

female, $n=10$, average age 22 years, mean weight $70.5 \mathrm{kgs}$, all right hand dominant) actively involved in overhead sports and/or sports reliant on GHJ rotation were included in the study. Sports included were: Tennis, Badminton, Netball, Volleyball, Basketball, Lacrosse, Squash, Cricket Bowling and Golf. Inclusion criteria were: both gender, aged over 16 years, training at least once a week during the season, club/ recreational level. Each participant was required to attend for 2 hours with a minimum of 48 hours between testing sessions. Prospective participants with a history of injury to the shoulder complex of the dominant arm within the last two years were excluded. Arm dominance was assessed as the arm they would chose to throw a ball or hold a racket [10]. The study received ethical approval from the department of sport and physical activity ethics committee at Edge Hill University in accordance with the declaration of Helsinki.

\subsection{Procedure}

An experimental design with three (neoprene sleeve, $\mathrm{KT}$, control) $\times 2$ (pre and post exercise) repeated measures was performed to investigate the Gleno-humeral joint (GHJ) active reproduction of passive positioning (ARPP) absolute error scores for JPS of external rotation (ER) and internal rotation (IR) of the dominant arm. Joint position sense error scores were recorded across two time periods (pre and post exercise). The internal goniometer of the isokinetic dynamometer (IKD) measured the difference between the target position angle and the angle reproduced by the participant during ARPP.

\subsection{Measurements}

\subsubsection{Starting test positions}

Participants were required to attend on a total of three occasions to undertake each of the testing conditions. Three different GHJ positions were analysed consecutively with a 15 minute recovery period between each position [11]. These positions were assessed creating an extensive profile of shoulder function. The starting positions were: 9090 (sitting with $90^{\circ}$ of shoulder abduction and $90^{\circ}$ of elbow flexion [12], modified neutral (sitting with $45^{\circ}$ of shoulder abduction and $90^{\circ}$ of elbow flexion in the scapular plane [13] and diagonal (sitting performing flexion, abduction and external rotation and extension, adduction and internal rotation (IR) [14]. 


\subsubsection{Isokinetic proprioception testing}

Testing was performed on an isokinetic dynamometer (IKD), version 3 (Biodex Inc, Shirley, New York) in the seated position with straps applied in accordance with manufacturer's guidelines. Active relocation of passive positioning involved the subjects being blindfolded to avoid visual cues [9]. Gravity correction was employed with the GHJ in neutral rotation for each position to allow normalisation of limb weight at a point perpendicular to the floor $[10,15]$. Shoulder JPS absolute error scores were recorded by taking the mean of three pre-exercise repetitions and the mean of three post-exercise repetitions calculated by the internal goniometer of the IKD. Testing speed for all ARPP tests was $10^{\circ}$ /s $[9,16-18]$.

\subsubsection{Target angles}

Three different angles were included to provide error scores for end of range external rotation (ER), mid-range and end of range internal rotation (IR) assessment at each position thus assessing all potential mechanoreceptors involved in JPS [19]. At the 9090 position the entire ROM was $120^{\circ}$ The start position $\left(0^{\circ}\right)$ was $90^{\circ} \mathrm{GHJ}$ abduction, $90^{\circ}$ elbow flexion and neutral rotation. Internal rotation range of motion was $50^{\circ}$ from the start position and external rotation was $70^{\circ}$ from the start position. The IR angle was $40^{\circ}$ from the start position, ER angle was $60^{\circ}$ from the start position and the mid-range positon was $30^{\circ}$ from neutral into ER. At the modified neutral position the entire $\mathrm{ROM}$ was $100^{\circ}$ The start position $\left(0^{\circ}\right.$ was $45^{\circ} \mathrm{GHJ}$ abduction in the frontal plane. Internal rotation (IR) range of motion was $40^{\circ}$ from the start position and external rotation was $60^{\circ}$ form the start position. The IR angle was $30^{\circ}$ from the start position, ER angle was $50^{\circ}$ from the start position and the mid-range positon was $50^{\circ}$ from neutral into ER. At the diagonal position the entire ROM was $90^{\circ}$ End of range IR was $0^{\circ}$ with end of range ER $90^{\circ}$ Internal rotation joint angle was $10^{\circ}$ from end of range ER, external rotation was $10^{\circ}$ from end of range IR and the mid-range angle was $45^{\circ}$ from end of range ER. The testing procedures were counterbalanced for control, neoprene and KT for each participant. The order of positioning i.e. 9090, modified neutral and diagonal was not counter-balanced in order to create a functionally representative protocol relating to the battery of sports included.

\subsubsection{Isokinetic exercise protocol}

A 30 repetition isokinetic exercise protocol [20] at $120 \%$ s [16] acted as the exercise component. A pilot study determined that a 30 repetition isokinetic protocol was not sufficient to induce fatigue and subsequently fatigue was not a variable under scrutiny. The protocol employed represented an association with time duration thus simulating game play. Cardiovascular and metabolic responses are significantly developed after isokinetic endurance training [21]. As such the 30 repetition protocol is a valid means of administering resistance capable of representing endurance training observed in game play [22]. Participants were asked to provide maximal effort throughout the entire ROM and exercise until the researcher told them to stop. The protocol carried out was identical to the position being tested i.e. 9090 as specified above. A 15 minute rest interval [11] allowing recovery time mimicking such activities which have intervals and breaks throughou game situations, was provided.

\subsection{Supportive devices}

\subsubsection{Kinesio-tape $(K T)$}

The kinesio-tape used in this study was Rock Tape [23]. Application was in accordance with manufacturer's guidelines for a shoulder throwing taping. This technique was chosen as it most closely adhered to the functional requirements of the overhead sports included. The manufacturer [23] suggests application of this type of taping be applied along fascial lines whilst the fascia is on stretch. Application of the tape was performed with the dominant arm in a stretched position. An anterior strip of tape was applied from the wrist travelling upwards across the forearm flexor muscles, Biceps Brachii, across the Pectoralis Major muscle finishing before the sternum. A posterior strip of tape was applied in the same manner from the wrist upwards across the forearm extensor muscles, Triceps Brachii, Posterior Deltoid, and Upper Fibres of Trapezius, finishing at the lower cervical vertebrae. The tape was applied with no stretch, and can be seen in Fig. 1.

\subsubsection{Neoprene shoulder sleeve}

The neoprene sleeve (Fig. 2) was a non-branded sleeve and dependent on the size of the participant was either a size small/medium or large/extra-large. The sleeve utilised Velcro both anteriorly and posteriorly across the chest and superior torso respectively.

\subsection{Statistical analysis}

Statistical analyses and analysis parameters were determined a priori. The assumptions associated with a 


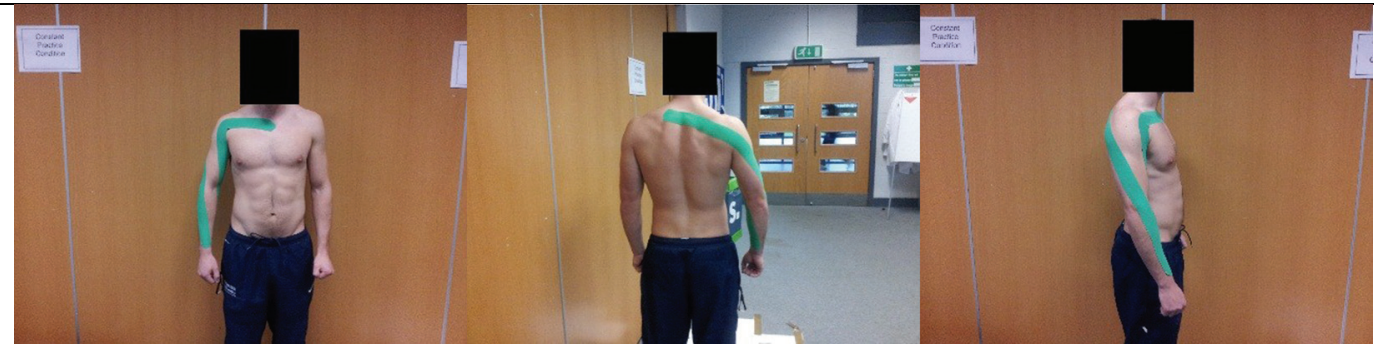

Fig. 1. Photograph of kinesiology tape application for shoulder throwing.

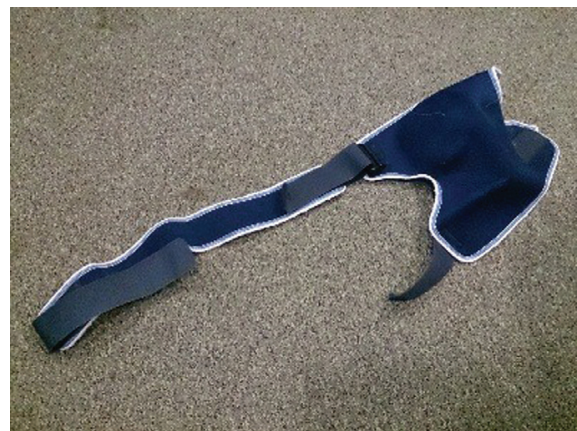

Fig. 2. Photograph of neoprene sleeve.

repeated measures general linear model (GLM) were assessed to ensure appropriateness, including consideration of residual normality and error of variance. Mauchly's test of sphericity was applied with a Greenhouse Geisser correction where appropriate. With no violation of assumptions, inferential analyses were performed using a mixed method two-way (exercise $*$ support) repeated measures GLM to examine differences pre- and post-exercise, and between support conditions with gender pooling performed. Preliminary analyses using a three-way (exercise $*$ support $*$ gender) GLM revealed no main effect for gender, and no interaction between gender and exercise or support. Subsequently all data was pooled for gender to provide an experimental data set of $n=25$. Post-hoc least square difference applications were applied where a significant main effect was observed. All statistical analyses were performed using PASW Statistics Editor 20.0 for windows (SPSS Inc, Chicago, USA). Statistical significance was set at $p \leqslant 0.05$.

\section{Results}

\subsection{0 position}

Figure 3 summarises the influence of exercise and support condition on the mean absolute joint position sense error scores at the 9090 position. There was no significant main effect for exercise in end of range internal rotation $(p=0.22)$, mid-range ( $p=0.15)$, or end of range external rotation $(p=0.51)$. Post-hoc analyses of support main effects identified a significant $(p<$ $0.01)$ impairment in pre-exercise external rotation JPS when KT was applied $\left(5.30 \pm 2.16^{\circ}\right)$ relative to the baseline control condition $\left(4.11 \pm 2.81^{\circ}\right)$. In external rotation, there was also a trend $(p=0.09)$ toward impaired performance in the neoprene sleeve condition $\left(4.15 \pm 3.34^{\circ}\right)$ relative to baseline control. At the midrange position there was a trend toward impaired performance post-exercise in the KT trial $\left(5.11 \pm 2.64^{\circ}\right)$ relative to both control $\left(3.91 \pm 2.20^{\circ} ; p=0.07\right)$ and neoprene $\left(4.19 \pm 2.25^{\circ} ; p=0.10\right)$ conditions.

\subsection{Modified neutral position}

Figure 4 summarises the influence of exercise and support condition on the mean absolute joint position sense error scores at the modified journal position. There was no significant main effect for exercise in end of range internal rotation $(p=0.59)$, mid-range ( $p=$ $0.38)$, or end of range external rotation $(p=0.27)$. There was no significant main effect identified for support condition, but post-hoc analyses of support main effects identified a trend $(p=0.09)$ toward impaired performance post-exercise in external rotation JPS for the neoprene trial $\left(5.76 \pm 1.94^{\circ}\right)$ relative to the preexercise scores $\left(4.55 \pm 2.42^{\circ}\right)$.

\subsection{Diagonal position}

Figure 5 summarises the influence of exercise and support condition on the mean absolute joint position sense error scores at the diagonal position. There was no significant main effect for exercise in end of range internal rotation $(p=0.16)$, mid-range $(p=0.11)$, or end of range external rotation ( $p=0.55)$. There was no significant main effect identified for support condi- 


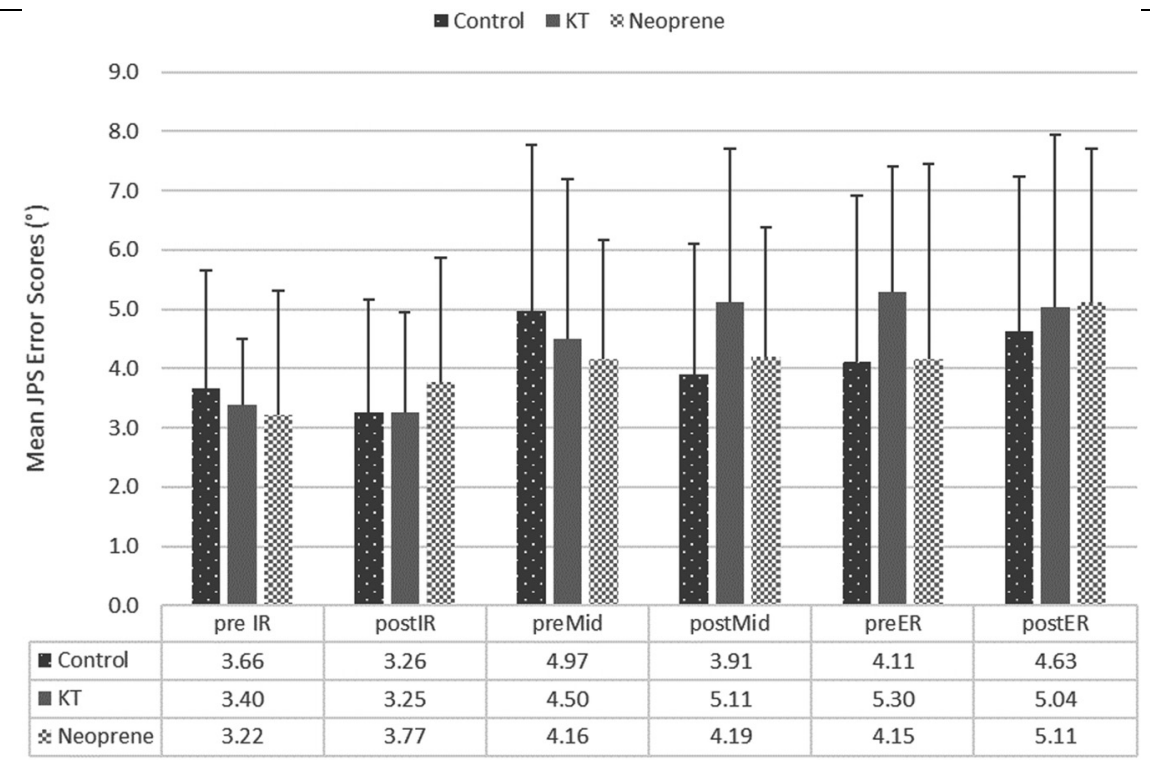

Fig. 3. The influence of joint support on JPS error scores in the 9090 position.

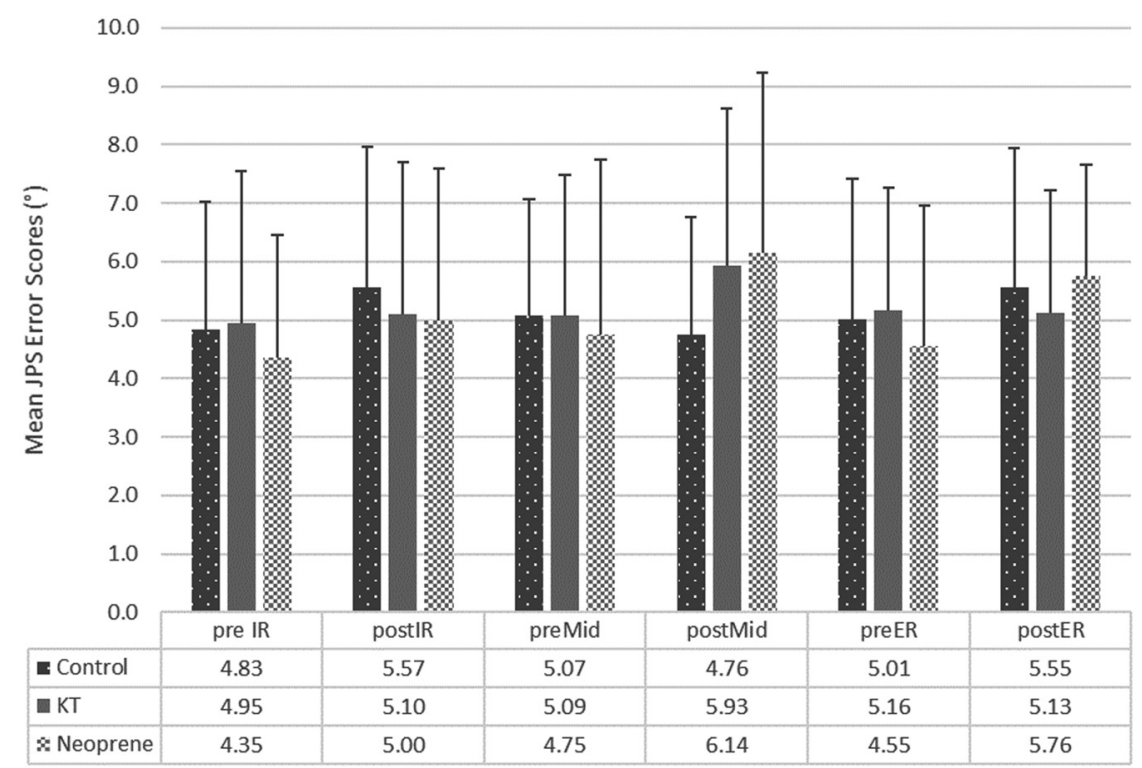

Fig. 4. The influence of joint support on JPS error scores in the modified neutral position.

tion, but post-hoc analyses identified a trend ( $p=0.09$ ) toward enhanced performance pre-exercise in external rotation JPS for the neoprene trial $\left(3.15 \pm 2.10^{\circ}\right)$ relative to the baseline control $\left(3.87 \pm 1.97^{\circ}\right)$.

\section{Discussion}

The aim of this study was to quantify the efficacy of functional shoulder supports in mediating the impact of exercise on joint position sense accuracy. The main findings of this study when assessing the mediating effects of kinesiology tape (KT) suggest that the application of KT pre-exercise created significantly poorer joint position sense error scores when compared to the control condition $(P<0.01)$ at end of range external rotation (ER) 9090 position. There was also a trend towards KT being less effective than both the neoprene sleeve and control at the mid-range 9090 position. Fur- 
6 L.M.Wilson and M. Greig / The efficacy of functional supports in mediating the effects of exercise on shoulder IPS

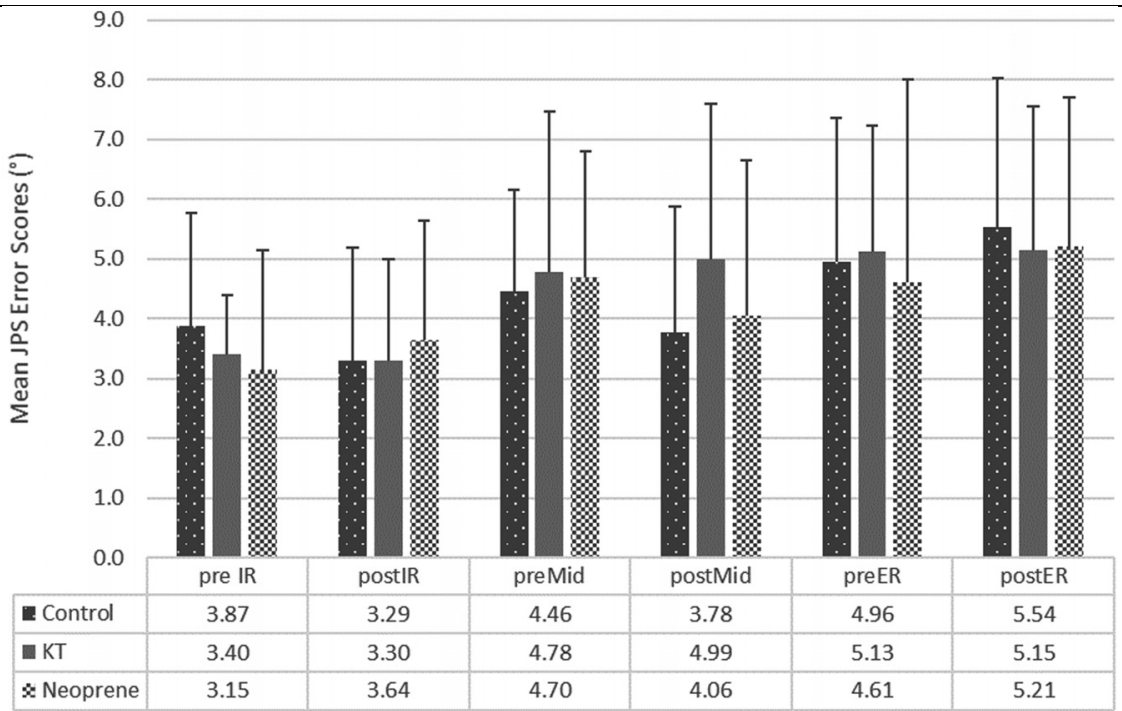

Fig. 5. The influence of joint support on JPS error scores in the diagonal position.

thermore, KT had no influence in comparison to the neoprene sleeve or control at the modified neutral or diagonal positions as no significant difference was determined at any angle. The effects of mechanical deformation related to stretch and compression [24] may suggests an association to capsular and fascial receptors such as Ruffini nerve endings and Pascinian corpuscles [25,26]. However, KT did not mediate exercise at any position at the mid-range. It has been reported that at $90^{\circ}$ of shoulder elevation KT diminished JPS acuity but at $50^{\circ}$ did not [27] therefore suggesting joint angles are an important factor to consider.

The neoprene sleeve appeared to be effected by both position and angle. At the 9090 position neoprene sleeve out-performed KT at the mid-range $(p=0.07)$ post-exercise suggesting that KT may impair function at this range. In comparison to the control condition of no tape, the neoprene sleeve had a trend towards being more effective at the diagonal position at the internal rotation (IR) angle ( $p=0.09)$ pre-exercise, however, this was not found with the 9090 position where the neoprene sleeve was less effective post-exercise at the ER angle $(p=0.09)$. The difference in findings between studies might be attributed to methodological differences, for example not using an overhead sports population and different application of tape (y strips) when applying KT [27]. Our hypothesis for the positive JPS acuity at EOR IR and the detrimental effects at mid-range (KT) may be explained by considering a lack of stretch on the fascia mid-range as an inability for tape to provide as great a stimulus to the underlying tissue and muscle receptors [28]. At a mid- range position muscular mechanoreceptor response is decreased [5]. This includes involvement of the Golgi tendon organ (GTO) and stretch reflex. Thus, it could be surmised that a different points in the range JPS acuity may alter. At the modified neutral position postexercise the neoprene sleeve showed a trend towards being worse than at pre-exercise but only at the ER angle. This may suggest the stimulus provided by the neoprene sleeve may begin to become inhibitive as exercise progresses. Eight to ten participants stated that they felt the neoprene sleeve and KT were restrictive when moving in to EOR positions. Skin stimulation from cutaneous receptors [29], in this instance, may have actually have been unhelpful with particular attention paid to the movement pattern in the modified neutral position as increased kinaesthetic stimulation may have over-stimulated at EOR causing JPS to alter.

The results suggest that joint position sense error scores were effected by position, angle and exercise state. There was little difference between the neoprene sleeve and KT in comparison to no tape. The population studied were healthy overhead athletes and the results may indicate that those with perceived acceptable joint position acuity may not benefit from the application of supportive devices, indeed, these may be detrimental to performance both in pre and post exercise states. The lower IR error scores produced when the neoprene sleeve was applied at the diagonal position and the lower mid-range scores at the 9090 position may be attributed to the compressive effect of the sleeve [30] and the effect on muscle and skin receptors [31]. A greater stimulus of the GTO and mus- 


\begin{abstract}
cle spindles at the mid-range position has previously been activated through stimulation of underlying muscle tissue from skin receptors being initially stimulated [32]. Nonetheless, this does not explain the lower error scores only at mid-range and IR range and not seen at the modified neutral position.

With the type of JPS being assessed (ARPP) mechanoreceptors involved more in active relocation where the participant actively guides the arm into position, may be affected more than if the relocation was done passively. Passive repositioning [33] may cause a decreased response after exercise from end of range structures such as the joint mechanoreceptors. Stretch may be more pertinent than compression with passive movements. To provide greater mechanistic understanding of the types of supportive device used in the present study, further research might consider the experimental applications provided by electromyography and medical imaging. The non-significant change in JPS over the duration of the exercise protocol might also be attributed to conflicting mechanisms associated with potentiation and fatigue. No warm-up was used as this would affect the mechanoreceptors within the GHJ and surrounding rotator cuff musculature when JPS was being [16]. The potentiating influence of a warm-up on JPS [34] might be evident over the initial stages of the exercise protocol, masking the expected exercise-effect $[33,35]$ in the latter stages. The population studied in the current research were overhead sports participants who repeat rotational GHJ movements many times during the course of play. This type of repetition may have caused adaptation to sports specific demands of play thus creating an increase in stimulus through compression and stretch [3].
\end{abstract}

\section{Clinical and practical implications}

Clinically, JPS acuity in the gleno-humeral joint may be sufficient without the addition of supportive devices. The current study utilised participants across a range of sports, and it is acknowledged that whilst movement patterns are similar between sports [36] muscle fibre recruitment and fatigue resistance may be different between sports such as tennis and volleyball (speed over power). The participants used were injury free, and as such the use of the supportive devices is directed toward injury prevention rather than injury management. When examining knee proprioception, participants with lower JPS acuity responded more effectively to KT than those with perceived good acu- ity [37]. As such, the results of the present study cannot be generalised to injured athletes, where an intervention could potentially be more effective. The 30 repetition protocol was sufficient to recreate the demands of overhead sports but did not induce a fatigue effect. Therefore, the results cannot be generalised to absolute fatigue conditions.

\section{Conclusion}

Further study is required on the functional kinesiology taping applied in this study as there is limited research supporting or refuting its use in sport. Neoprene shoulder sleeves are a less expensive and more user friendly support intervention, and as such, the minimal difference between KT, neoprene and no support may not warrant changing training or playing habits significantly in healthy overhead sports athletes. Indeed, KT impaired function at mid-range post-exercise and EOR pre-exercise at the 9090 position suggesting that healthy, uninjured overhead athletes may not need to consider taping or supportive device. The 9090 position is one in which several overhead sports require power and position sense together. Therefore, future research may consider the application in a group of subjects who have poor joint position acuity. Furthermore, the effects of fatigue have been more widely investigated in the GHJ $[33,38,39]$ and it may be pertinent to examine supportive devices under these conditions.

\section{Conflict of interest}

There are no financial or other potential conflicts of interest associated with this research.

\section{References}

[1] Teyhen DS, Miller JM, Middag TR and Kane EJ. Rotator cuff fatigue and glenohumeral kinematics in participants without shoulder dysfunction. J Athl Train. 2008; 4: 352-358.

[2] Kablan NF, Ertan H, Ünver F, Kirazcia K and Korkusuza F Factors affecting the shoulder proprioception sense in male volleyball players. Isokinet Exerc Sci. 2004; 12: 193-198.

[3] Sterner RL, Pincivero EM and Lephart SM. The effects of muscular fatigue on shoulder proprioception. Clin J Sport Med. 1999; 8: 96-101.

[4] Chen S-K, Simonian PT, Wickiewicz TD, Otis JC and Warren RF. Radiographic evaluation of glenohumeral kinematics: A muscle fatigue model. J Shoulder Elbow Surg. 1999; 8: 4952. 
[5] Janwantanakul P, Magarey ME, Jones MA and Dansie BR. Variation in shoulder position sense at mid and extreme range of motion. Arch Phys Med Rehabil. 2002; 82(6): 840-844.

[6] Ulker B, Kunduracioglu B, Cetin C and Güner RS. Effect of positioning and bracing on passive position sense of shoulder joint. Br J Sports Med. 2002; 38: 549-552.

[7] Chu JC, Kane EJ, Arnold BL and Gansneder BM. The effect of a neoprene shoulder stabilizer on active joint-reposition sense in subjects with stable and unstable shoulders. J Athl Train. 2002; 37(2): 141-145.

[8] Halseth T, McChesney JW, DeBeliso M, Vaughn R and Lien $\mathrm{J}$. The effects of kinesio taping on proprioception at the ankle. J Sports Sci Med. 2004; 3: 1-7.

[9] Nocera J, Rubley M, Holcomb W and Guadagnoli M. The effects of repetitive throwing on shoulder proprioception and internal and external rotation strength. J Sport Rehabil. 2006; 15: 351-362.

10] Zanca GG, Oliveira AB, Saccol MF and Mattiello-Rosa SM. Functional torque ratios and torque curve analysis of shoulder rotations in overhead athletes with and without impingement symptoms. J Sports Sci. 2001; 29(15): 1603-1611.

11] Parcell AC, Sawyer RD, Tricoli VA and Chinevere TD. Minimum rest period for strength recovery during a common isokinetic testing protocol. Med Sport Sci. 2002; 34(6): 10181022.

[12] Andrade M, Barbosa de Lira CA, Vancini RL, Aparecido de Almeida A, Amélia Benedito-Silva A and da Silva AC. Profiling the isokinetic shoulder rotator muscle strength in 13 to 36-year-old male and female handball players. Phys Ther Sport. 2013; 14: 246-252.

13] Edouard P, Degache F, Oullion R, Plessis J-Y, GleizesCervera $S$ and Calmels P. Shoulder strength imbalances as injury risk in handball. Int J Sports Med. 2013; 34: 654-660.

14] Baltaci $G$ and Tunay VB. Isokinetic performance at diagonal pattern and shoulder mobility in elite overhead athletes. Scand J Med Sci Sports. 2004; 14: 231-238.

15] Mayer F, Horstmann T, Baurlea W, Grau S, Handel M and Dickhuth HH. Diagnostics with isokinetic devices in shoulder measurements - potentials and limits. Isokinet Exerc Sci. 2001; 9: 19-25.

16] Mota N and Ribeiro F. Association between shoulder proprioception and muscle strength in water polo players. Isokinet Exerc Sci. 2012; 20: 17-21.

[17] Julienne R, Gauthier A and Davenne D. Fatigue-resistance of the internal rotator muscles in the tennis player's shoulder Isokinetic and electromyographic analysis. Phys Ther Sport. 2012; 13: 22-26.

18] Forthomme B, Dvir Z, Crielaard JM and Croisier JL. Isokinetic assessment of the shoulder rotators: A study of optimal test position. Clin Physiol Funct I. 2011; 31: 227-232.

[19] Myers JB, Wassinger CA and Lephart SM. Sensorimotor contribution to shoulder stability: Effect of injury and rehabilitation. Man Ther. 2006; 11: 197-201.

20] Ellenbecker TS and Roetert EP. Testing isokinetic muscular fatigue of shoulder internal and external rotation in elite junior tennis players. J Orthop Sports Phys Ther. 1999; 29(5): 275281.

[21] Edouard P, Castells J, Calmels P, Roche F and Degache 503
F. Cardiovascular and metabolic responses during isokinetic shoulder rotators strength testing in healthy subjects. Isokinet Exerc Sci. 2010; 18: 23-29.

[22] Kovacs MN. Applied physiology of tennis performance. Br J Sports Med. 2006; 40: 381-386

[23] Rocktape Inc, USA (Cited 2014 August 11th 2014) Available from: www.rocktape.com.

[24] Voight ML, Harden AJ, Blackburn TA, Tippett S and Canner GC. The effects of muscle fatigue on and the relationship of arm dominance in shoulder proprioception. J Orthop Sports Phys Ther. 1996; 23(6): 348-352.

[25] Simmonds N, Miller P and Gemmell H. A theoretical framework for the role of fascia in manual therapy. J Bodyw Mov Ther. 2012; 16: 83-93.

[26] Grigg P. Peripheral neural mechanisms in proprioception. Sports Rehabil. 1994; 3: 2-17.

[27] Aarseth LM, Suprak DN, Chalmers GR, Lyon L and Dahlquist DT. Kinesio tape and shoulder-joint position sense. J Athl Train. 2015; 50(8): 785-791.

[28] Ljubisavljevicu M, Vukcevic IS, Radovanovic S, Milanovic $\mathrm{S}$ and Anastasijevic R. Effects of cutaneous afferent input on fatigue-induced changes in fusimotor activity of decerebrate cats. Neuroscience. 1997; 79(3): 935-942.

[29] Collins DF, Refshauge KM, Todd G and Gandevia SC. Cutaneous receptors contribute to kinesthesia at the index finger elbow, and knee. J Neurophysiol. 2005; 94(3): 1699-1706.

[30] Herrington L, Simmonds S and Hatcher J. The effects of a neoprene sleeve on knee joint position sense. Res Sports Med 2005; 13: 37-46.

[31] Riemann BL and Lephart SM. The sensorimotor system, par I: Physiologic basis of functional joint stability. J Athl Train 2002; 37(1): 71-79.

[32] Carpenter JE, Blasier RB and Pellizzon GB. The Effects of Muscle Fatigue on Shoulder Joint Position Sense. Am J Sports Med. 1998; 26(2): 262-265.

[33] Chang H-Y, Chen C-S, Wei S-H and Huang C-H. Recovery of joint position sense in the shoulder after muscle fatigue. Sports Rehabil. 2006; 15: 312-315.

[34] Magelhaes T, Ribeiro F, Pineiro A and Oliveiro J. Warming up before sporting activity improves knee position sense. Phys Ther Sport. 2010; 11: 86-90.

[35] Murray TA, Cook TD, Werner LS, Schlegel TS and Hawkins RJ. The effects of extended play on professional basebal pitchers. Am J Sports Med. 2001; 29(2): 137-142.

[36] Van der Hoeven H and Kibler WB. Shoulder Injuries in Tenni Players' Br J Sports Med. 2006; 40: 435-440.

[37] Hosp S, Bottonia G, Heinrich D, Kofler P, Hasler M and Nachbauer W. A pilot study of the effect of Kinesiology tape on knee proprioception after physical activity in healthy women. J Sci Med Sport. 2015; 18: 709-713.

[38] Teyhen DS, Miller JM, Middag TR and Kane EJ. Rotator cuf fatigue and glenohumeral kinematics in participants withou shoulder dysfunction. J Athl Train. 2008; 4: 352-358.

[39] Bowman TG, Hart JM, McGuire BA, Palmieri RM and Inger soll CD. A functional fatiguing protocol and deceleration time of the shoulder from an internal rotation pertubation. J Ath Train. 2006; 41(3): 275-279. 